\title{
Desarrollo y evaluación de la competencia transversal 'Creatividad, innovación y emprendimiento' en la asignatura 'Explotación del transporte aéreo y organización aeronáutica'.
}

\section{José María Mateu ${ }^{a}$}

${ }^{a}$ Departamento de ingeniería e infraestructura de los transportes de la Universitat Politècnica de València (UPV), jomaces1@tra.upv.es.

\begin{abstract}
'Creativity, innovation and entrepreneurship' is a competence included into its transversal competences by the Polytechnic University of Valencia. It is an essential competence for the development of the students, as well as for their engagement in the society with the role they deserve for their qualifications. The development of this competence must be embedded in the development of other more technical competences, to ensure that they are properly internalized and added to the students' automatic reasoning mechanisms. We show an experience in this line, consisting in a classroom practice designed to develop the competence under the subject of 'Air transport exploitation and aeronautical organization', in the aeronautical studies. The practice includes the co-assessment by peers.
\end{abstract}

Keywords: creativity, innovation, entrepreneurship, competences, assessment, air transport, business model, lateral thinking.

\begin{abstract}
Resumen
La competencia 'Creatividad, innovación y emprendimiento', incluida por la Universitat Politècnica de València en su lista de competencias transversales, es una competencia esencial en el desarrollo de los estudiantes, y en su preparación para asumir el rol que como titulados superiores la sociedad les asigna. El desarrollo de la competencia debe estar enmarcado en el desarrollo de las demás competencias de carácter más técnico, como vía para garantizar que el estudiante la interioriza y la añade a sus mecanismos automáticos de razonamiento. Presentamos una experiencia en esta línea, consistente en una Práctica que desarrolla la competencia en el curso de la asignatura 'Explotación del transporte aéreo y organización aeronáutica', de los estudios de Ingeniería aeronáutica. La Práctica incorpora además la evaluación de la misma a cargo de los compañeros y compañeras de los y las estudiantes (co-evaluación).
\end{abstract}

Palabras clave: creatividad, innovación, emprendimiento, competencias, evaluación, transporte aéreo, modelo de negocio, pensamiento lateral. 


\section{1.- Introducción}

El Espacio Europeo de Educación Superior, con la Universidad como principal referente, constituye en la actualidad el principal agente de la planificación e impartición de los procesos formativos de mayor nivel en Europa. En la medida en que no existe un nivel superior, las exigencias sobre el ámbito universitario son elevadas, puesto que sus egresados y egresadas acceden directamente a puestos de responsabilidad en las instituciones que configuran la sociedad real. Deben estar pues preparados para ello, deben de estar en condiciones de asumir los roles que la sociedad les confía.

Desde hace unas décadas se sostiene incluso la conveniencia de que la Universidad asuma de manera más directa la tarea de crear oportunidades, la creación de empleo y riqueza. Es la denominada 'tercera misión', que se añade a las dos tradicionales: la creación de conocimiento, o investigación, y su transmisión, o docencia (Martin y Etzkowitz, 2000).

Algunos van más allá y enmarcan en el relativamente nuevo ámbito de la Responsabilidad Social Universitaria la responsabilidad cívica que empuja a las universidades a convertirse en agentes de cambio social (Naval et al., 2011).

Con mayor o menor decisión, las Universidades han tomado iniciativas en este sentido, creando parques científicos, dando soporte a empresas spin-off, promoviendo acuerdos de investigación con empresas, comercializando los resultados de la investigación desarrollada, etc. El éxito ha sido desigual, dependiendo además de las expectativas previstas, el esfuerzo desplegado y los recursos involucrados.

En todo caso, todas estas medidas sirven preferentemente para apoyar las iniciativas personales de los miembros más activos de la comunidad universitaria, no para promover un cambio social que generalice la actitud emprendedora entre los distintos colectivos universitarios. Hay que recordar que sólo el $5,7 \%$ de la población adulta española está involucrada en actividades emprendedoras en fase inicial, algo que apenas ha variado a lo largo de los años (Peña, Guerrero y González-Pernía, 2015).

La generalización del espíritu emprendedor requiere de un esfuerzo formativo y cultural de mayor alcance, un esfuerzo que embeba la actitud emprendedora en toda la actividad desarrollada en la universidad, o al menos en una buena parte de ella. La introducción de competencias transversales en los planes de estudio de nuestras universidades es en este sentido una buena noticia, porque construye y avanza en esta dirección.

La Universitat Politècnica de València, en particular, ha concretado en 13 las competencias transversales que todos sus estudiantes deberían adquirir a lo largo de su paso por la Universidad, incluyendo entre ellas la competencia denominada 'Innovación, creatividad y emprendimiento'. Se trata de una competencia que no se venía trabajando en los estudios de ingeniería, por lo que su introducción requiere en sí misma de una clara convicción por parte del personal docente, y una firme voluntad de acometer el cambio.

Algunas propuestas precursoras, desarrolladas en otros estudios y especialidades, nos sirven de inspiración y guía (López, 2016; Marin-Garcia, Ramirez y Andreu, 2015). Más próxima a nuestra propuesta está la de Ruiz y otros (2015), que aplica herramientas creativas

(cc) EY-NC-ND 2017, Universitat Politècnica de València 
(Design Thinking) en busca de nuevos modelos de negocio en un contexto genérico (no directamente relacionado con la especialidad de los estudiantes), empleando también instrumentos de evaluación (rúbricas) de carácter genérico.

Nuestra experiencia avanza sobre éstas al menos en tres direcciones:

1. proponiendo una Práctica que embebe la innovación en la especialidad concreta que cursan los estudiantes,

2. empleando instrumentos de evaluación específicos para medir el potencial de la innovación en modelos de negocio desplegada por los estudiantes, y

3. poniendo esa evaluación en manos de los compañeros y compañeras de los que han propuesto los nuevos modelos (co-evaluación).

La experiencia se enmarca en la asignatura 'Explotación del transporte aéreo y organización aeronáutica', que forma parte del plan de estudios del Máster Universitario en Ingeniería Aeronáutica de la Universitat Politècnica de València, tratándose además de un Máster habilitante para el ejercicio de la profesión de ingeniería aeronáutica.

La asignatura ha sido escogida como contribuidora al desarrollo de dos de las trece competencias transversales, las de 'Pensamiento crítico' e 'Innovación, creatividad y emprendimiento'. La asignatura ha sido escogida además como punto de control para ambas competencias (una asignatura es punto de control de una competencia cuando no sólo tiene la misión de trabajar la competencia, sino que debe además evaluar el progreso del alumno o alumna en la competencia).

\section{2.- Objetivos}

El objetivo de este trabajo es mostrar una experiencia concreta, desarrollada en el contexto de la citada asignatura, que desarrolla y evalúa la competencia transversal 'Innovación, creatividad y emprendimiento'.

La acción que exponemos perseguía el objetivo comentado asumiendo ciertas exigencias autoimpuestas. Además del rigor y la actualización en los contenidos que el marco universitario presupone, se pretendía:

- Que los contenidos aportados estuvieran encuadrados en el contexto real de las necesidades de desarrollo de una empresa innovadora del sector del transporte aéreo.

- Que la parte más sustancial del aprendizaje se desarrollara de manera armónica en el curso de una actividad práctica que involucrara tanto la competencia transversal en cuestión como las competencias de carácter general desarrolladas por la asignatura, por ejemplo 'que los estudiantes sepan aplicar los conocimientos adquiridos y su capacidad de resolución de problemas en entornos nuevos o poco conocidos dentro de contextos más amplios (o multidisciplinares) relacionados con su área de estudio'.

- Que esa actividad práctica incorporara el desarrollo de otras competencias que en el mundo real van intrínsecamente entrelazadas al proceso creativo, innovador y 
emprendedor, como la 'comprensión e integración', el 'trabajo en equipo y liderazgo' y el 'pensamiento crítico'.

\section{3.- Desarrollo de la innovación}

La asignatura 'Explotación del transporte aéreo y organización aeronáutica' se encuadra en la agenda del segundo semestre del primer curso del 'Máster Universitario en Ingeniería Aeronáutica'. La Práctica que se describe fue propuesta y desarrollada por primera vez en el segundo semestre del curso 2015-2016, con el detalle y resultados que a continuación se describen.

\section{1.- Contenidos previos aportados por la asignatura}

El programa de la asignatura comienza por el estudio del marco institucional del transporte aéreo, para adentrarse a continuación en las claves para la explotación eficaz y eficiente de las distintas modalidades del mismo: transporte comercial de pasajeros, transporte comercial de mercancías, aviación corporativa o ejecutiva, otros trabajos aéreos, la asistencia aeroportuaria o handling, etc.

En lo relativo al apartado de la Innovación el programa incorpora aspectos como 'los roles que puede jugar la innovación en la empresa de transporte aéreo', 'los ámbitos de aplicación de la misma' y, con especial énfasis, el aspecto de 'la innovación en modelos de negocio en el transporte aéreo'.

De relativamente reciente aparición, la innovación en modelos de negocio ha recibido una notable atención a lo largo de los años transcurridos de este siglo, tanto entre académicos de la Dirección de empresas como entre directivos y consultores. Este significativo interés en el concepto deviene del potencial teórico y práctico del mismo: "La innovación en modelos de negocio ha reconfigurado industrias enteras y ha redistribuido valor por miles de millones de dólares" (Johnson, Christensen y Kagermann, 2008; pág. 52). Las expectativas depositada en él son aún mayores: "Una empresa tiene tanto que ganar desarrollando un innovador modelo de negocio nuevo como desarrollando una innovadora nueva tecnología" (Chesbrough, 2010; pág. 356).

El ámbito del transporte aéreo se postula además como un ámbito donde la innovación en modelos de negocio ha dado y puede producir en el futuro importantes resultados. La literatura interpreta como innovaciones en modelos de negocio dos ejemplos paradigmáticos que han supuesto saltos cualitativos de alcance en sus respectivos mercados:

- la introducción de la 'aviación de bajo coste', en el mercado de transporte aéreo de pasajeros (Casadesus-Masanell y Ricart, 2010).

- la irrupción en el mercado de transporte aéreo de mercancías de los denominados 'integradores', empresas que asumen todos los pasos en una cadena de valor antes totalmente fragmentada (Shaw, 2007).

(cc) EY-NC-ND 2017, Universitat Politècnica de València 
Cuando estos ejemplos se proyectan al futuro, podemos concluir que "las innovaciones importantes no vendrán probablemente del vehículo de transporte, sino del sistema en el cual este vehículo se incorpora" (Crozet, 2010; pág. 20).

\section{2.- Planteamiento de la Práctica}

Siguiendo las directrices de Bolonia, se trataba entonces de configurar actividades en las que el alumno pudiera alcanzar los objetivos de aprendizaje de manera práctica, aplicada, partiendo de los contenidos explicitados en el apartado anterior.

La Práctica diseñada recibe por título 'Modelos de negocio en la aviación ejecutiva', y consiste en aplicar herramientas creativas de innovación en modelos de negocio al ámbito de esta modalidad de aviación, en busca de modelos de negocio innovadores y potencialmente capaces de crear servicios y mercados nuevos.

La aviación ejecutiva se define como la actividad de transportar grupos pequeños de personas (hasta 20) de manera segura, cómoda y eficiente en aeronaves apropiadas para ello. El modelo de negocio tradicional de la aviación ejecutiva consiste en el alquiler de la aeronave completa por parte de una empresa, institución o particular, durante un periodo de tiempo determinado. Ese periodo puede ser de horas, días, semanas o hasta años, y durante el mismo los clientes dispondrán de la aeronave y su tripulación con total dedicación y flexibilidad. Otros modelos de negocio han sido ensayados con mayor o menor fortuna. Entre los más utilizados la compra de la aeronave por el usuario (aviación privada), la compra compartida por varios usuarios (multipropiedad), la compartición del vuelo entre varias empresas o usuarios, etc.

La Práctica propone el uso de instrumentos creativos en busca de innovaciones en los modelos de negocio aplicables a la aviación ejecutiva. Los métodos creativos propuestos consisten concretamente en la aplicación del Pensamiento lateral (De Bono, 1967) a herramientas conceptuales habitualmente utilizadas en el ámbito del análisis e innovación en modelos de negocio, como es el caso del lienzo, o canvas, del modelo de negocio (Osterwalder y Pigneur, 2010) o la cadena de valor (Porter, 1985). Se trata de una metodología creativa ya empleada con éxito por el autor de este trabajo en el desarrollo de su Tesis doctoral (Mateu, 2015). En aquel caso se aplicaba a industrias más diversas (construcción, hostelería, fabricación cerámica, etc.).

La Práctica se estructura según la agenda detallada en la Tabla 1.

Tabla 1. Agenda de la Práctica

\begin{tabular}{|l|r|}
\hline Tarea & Duración aprox. \\
\hline $\begin{array}{l}\text { Presentación por el profesor del Pensamiento Lateral y la forma de } \\
\text { aplicarlo al Lienzo del modelo de negocio (ya expuesto en clase al }\end{array}$ & \multirow{2}{*}{ minutos } \\
hablar de Innovación en modelos de negocio), y a la cadena de valor. \\
Todo ello ilustrado con ejemplos de aplicación a otras modalidades de
\end{tabular}




\begin{tabular}{|l|r|}
\hline aviación y otros sectores. & 30 minutos \\
\hline $\begin{array}{l}\text { Trabajo en equipos de tres o cuatro estudiantes, aplicando lo anterior a } \\
\text { la búsqueda de nuevos modelos de negocio en el ámbito de la aviación } \\
\text { ejecutiva (parte principal de la Práctica) }\end{array}$ & 10 minutos \\
\hline $\begin{array}{l}\text { Elección del mejor Modelo de entre los encontrados por el Equipo y } \\
\text { preparación de la presentación del mismo }\end{array}$ & 5 minutos por \\
\hline $\begin{array}{l}\text { Presentación por el portavoz de cada Equipo de su nuevo Modelo al } \\
\text { resto de la clase }\end{array}$ & \\
\hline
\end{tabular}

\section{3.- Evaluación de los modelos}

Durante la exposición por cada equipo de su modelo, se instó a que escribieran en la pizarra los elementos esenciales y definitorios del mismo, de manera que quedaran todos ellos expuestos ante la clase, a modo de recordatorio para la posterior evaluación. La Figura 1 muestra el aspecto de la pizarra durante el desarrollo de la Práctica en una de las clases, una vez presentados tres de los modelos.

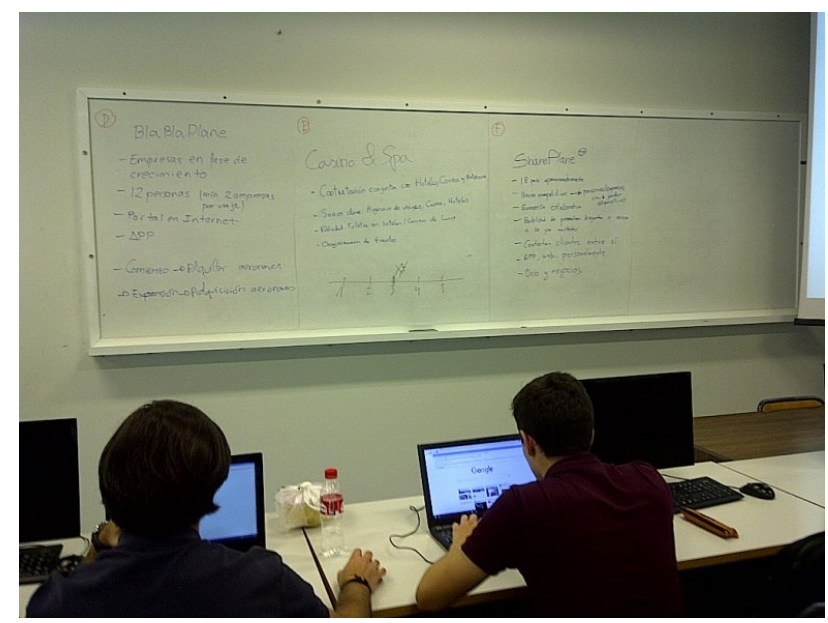

Fig. 1 Aspecto de la pizarra tras la presentación de tres de los modelos

La evaluación de los modelos fue acometida por los propios estudiantes, siguiendo las instrucciones facilitadas por el profesor. Primero se realizó una estimación meramente intuitiva, a efectos de ir introduciendo a los estudiantes en el proceso evaluador. A continuación se aplicó el verdadero 'Baremo de evaluación', desarrollado por el autor de esta comunicación en trabajos anteriores (Mateu, 2015; Mateu y March, 2016), y que se propuso a modo de rúbrica de evaluación (Moskal, 2000). Al tratarse de una rúbrica específica para modelos de negocio, su aplicación resulta más objetiva en nuestra opinión que otras rúbricas más generales propuestas para la evaluación de la innovación (por ejemplo la propuesta por Cuenca y otros (2015)).

(cc) EY-NC-ND 2017, Universitat Politècnica de València 
El Baremo de evaluación contempla ocho indicadores. Cada uno de ellos se evalúa mediante una escala de tipo Lickert de cinco posibles valores (1 a 5). Se facilitó a los estudiantes una plantilla con el detalle de los indicadores y una escala para que situaran en ella la letra identificadora de cada modelo. La mecánica consistió en que el profesor expusiera, indicador a indicador, lo que cada uno de ellos mide, dando tiempo a continuación para que los estudiantes valoraran cada modelo para ese indicador. La plantilla facilitada a los estudiantes incorporaba en cualquier caso la descripción de modelos merecedores de los valores extremos de cada indicador ( 1 y 5), así como ejemplos de modelos conocidos con valoraciones intermedias. Dicha plantilla, incorporando el Baremo de evaluación, se incluye como Anexo al presente documento.

La puntuación de cada modelo en cada indicador se obtuvo como media de las puntuaciones aportadas por los distintos estudiantes que lo evaluaron: todos los estudiantes de la clase en que se presentó, a excepción de los que habían diseñado el modelo evaluado (co-evaluación). La nota final del modelo se obtuvo como media de los ocho indicadores.

\section{4.- Aportación a la evaluación de la competencia}

La normativa de la UPV establece cuatro posibles calificaciones para las competencias transversales: desarrollo no alcanzado (D), en desarrollo (C), adecuado (B) y excelente (A).

Se consideró que el 'desarrollo no alcanzado' debería reservarse para modelos con valoración inferior a 2,5 y que las valoraciones superiores a 3,7 son difíciles de alcanzar, por lo que el Baremo propuesto fue:

- D: para valoraciones inferiores a 2,5

- $\quad$ : para valoraciones superiores a 2,5 e inferiores o iguales a 2,9

- B: para valoraciones superiores a 2,9 e inferiores o iguales a 3,3

- A: para valoraciones superiores a 3,3

Hay que tener en cuenta no obstante que la Práctica contribuía al desarrollo y evaluación de la competencia transversal, pero este desarrollo y sobre todo la evaluación se complementaba con otras acciones de carácter individual.

\section{4.- Resultados}

La Práctica fue planteada en los tres grupos de prácticas de la asignatura, involucrando un total de 56 estudiantes, que desarrollaron y presentaron un total de 17 modelos de negocio. Ello supone medias de 3,29 estudiantes por grupo y 5,67 equipos/modelos por clase.

Los estudiantes no evaluaban su propio modelo, pero sí los demás modelos presentados en su clase. Ello aportó una media de 15,53 evaluaciones para cada uno de los 17 modelos presentados.

Los modelos presentados exhibieron una casuística muy diversa en lo que a elementos innovadores se refiere. Resulta difícil catalogar esta casuística por cuanto que las 
innovaciones en modelos de negocio suelen afectar a varias partes del modelo. No obstante, y a efectos de tener una primera aproximación de los resultados, catalogaremos los modelos presentados en base al elemento innovador más característico del modelo propuesto. De este modo, y siguiendo la taxonomía de Osterwalder y Pigneur mostrada en la figura 2 (Osterwalder y Pigneur, 2010), podemos catalogar las innovaciones incorporadas a los modelos desarrollados en la Práctica en las siguientes categorías:

- Innovaciones basadas en cambios en la propuesta de valor mediante mejora de la experiencia en vuelo: entretenimiento a bordo (casino en vuelo), vistas exteriores (mediante ventanas panorámicas), descanso en vuelo (hotel a bordo),...

- Innovaciones basadas en cambios en la propuesta de valor tendentes a ofrecer otras utilidades: oportunidades de networking aprovechando el servicio,...

- Innovaciones basadas en la forma de ofrecer el vuelo (canales): incluido en paquetes turísticos de alto valor, a través de portales web y apps móviles, en el momento de perder un vuelo regular,...

- Innovaciones basadas en el tipo de aeronave utilizado (recurso clave): aeronaves capaces de transportar el automóvil a bordo, jets equipados con elementos de comodidad particulares, helicópteros,...

- Innovaciones basadas en fórmulas de precios bajos (flujo de ingresos): tarifa plana, paquetes de horas, fórmulas de tarificación especiales,...

- Innovaciones basadas en cambios más radicales del modelo tradicional: economía colaborativa (compartir avión), venta de asientos en rutas programadas,...

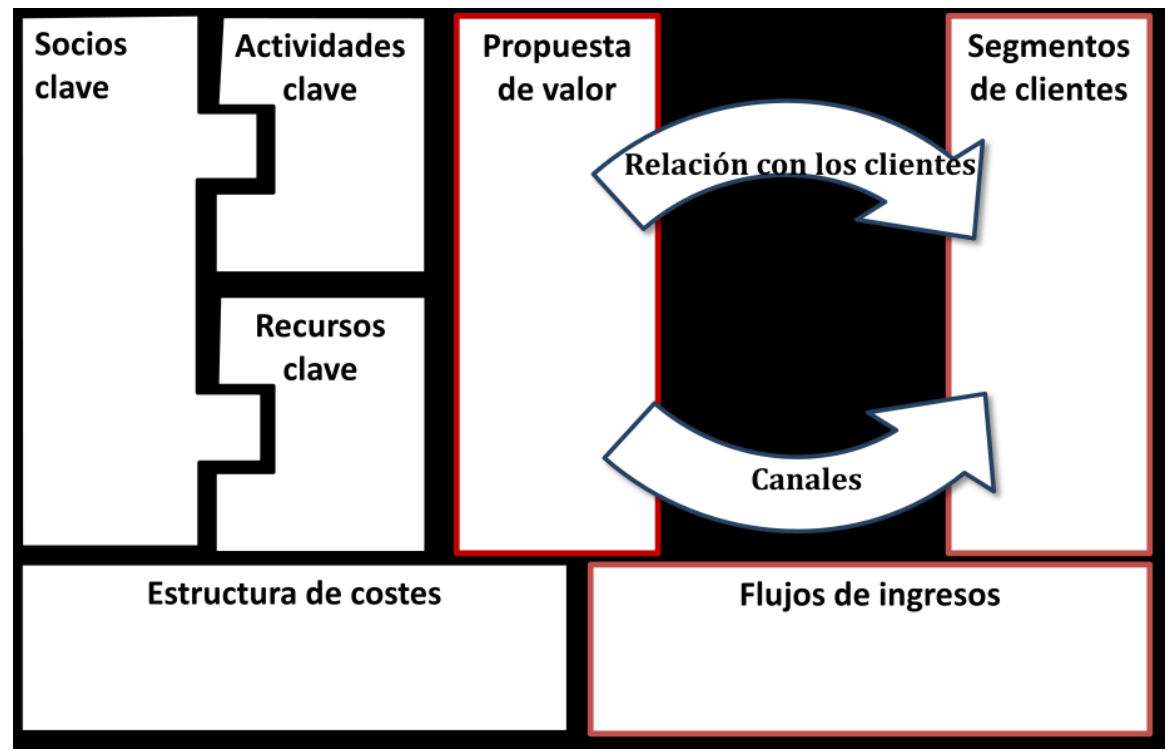

Fig.2 Lienzo del modelo de negocio (Osterwalder y Pigneur, 2010)

El modelo evaluado con menor nota obtuvo un 1,65 en el indicador peor evaluado, y un 3,41 en el mejor puntuado, para una nota media de los ocho indicadores de 2,50. Se trataba 
de un modelo que sustituía los jets ejecutivos por helicópteros, reduciendo el radio de acción a núcleos urbanos.

El modelo evaluado con mayor nota obtuvo un 2,50 en el indicador peor evaluado, y un 4,29 en el mejor puntuado, para una nota media de los ocho indicadores de 3,64. Se trataba de un modelo que aplicaba la lógica de la aviación de bajo coste a la ampliación de la base de clientes de la aviación ejecutiva.

La evaluación de los 17 modelos y de sus autores, derivada de esta Práctica, se ajustó a:

- ningún modelo evaluado con $\mathrm{D}$,

- 8 modelos evaluados con $\mathrm{C}$,

- 6 modelos evaluados con B

- y 3 modelos evaluados con A.

El comportamiento mostrado por los estudiantes durante la Práctica fue mayoritariamente de aplicación e interés. Se mostraron entretenidos y muy participativos.

No hubo objeciones, y se aceptó de buen grado, que las valoraciones de los compañeros (co-evaluación) sirvieran para la evaluación de la competencia, si bien hay que decir que los estudiantes no parecen tener excesivo conocimiento ni dar demasiada importancia a la evaluación de estas competencias.

\section{5.- Conclusiones}

Este trabajo se limita a mostrar una experiencia concreta de desarrollo y evaluación de una competencia como la 'creatividad, innovación y emprendedurismo' en el contexto de una asignatura de un plan de estudios de una Ingeniería. No obstante, y hasta donde esta experiencia pueda servir de ejemplo, se pueden extraer las siguientes conclusiones:

- El aprendizaje puede verse notablemente facilitado con el uso de actividades que combinen el desarrollo de competencias técnicas y transversales, en particular la competencia transversal en cuestión. El aprendizaje de ambos tipos de competencia se ve mutuamente reforzado.

- Esa combinación resulta además amena y estimulante para los estudiantes.

- La evaluación por los compañeros y compañeras (co-evaluación) de los resultados prácticos obtenidos es bien acogida por los estudiantes evaluados, al menos en la medida en que se base en una metodología clara y objetiva.

- La utilización de una rúbrica más específica facilita una co-evaluación más objetiva, lo que a la postre puede evitar los efectos indeseados identificados por algunos autores, como es el caso de evaluaciones "generalmente elevadas y con poca dispersión entre los distintos items" (Pérez-Zuriaga y otros, 2015, pág. 848).

- Lo más complicado, y en lo que habría que profundizar, sería el traslado de la evaluación de los resultados objetivos a la escala de evaluación de la competencia transversal. 
Desarrollo y evaluación de la competencia transversal 'Creatividad, innovación y emprendimiento' en la asignatura 'Explotación del transporte aéreo y organización aeronáutica'

\section{6.- Referencias}

CASADESUS-MASANELL, R., y RICART, J. E. (2010). "From Strategy to Business Models and onto Tactics " en Long Range Planning, vol. 43, issue 2-3, p. 195-215.

CHESBROUGH, H. (2010). "Business Model Innovation: Opportunities and barriers" en Long Range Planning, vol. 43, issue 2-3, p. 354-363.

CROZET, Y. (2010) "Driving forces of innovation in the transport sector". En: Transport and Innovation. Unleashing the potential. International Transport Forum (2010. Leipzig, Alemania). $\mathrm{OECD} / \mathrm{ITF}$.

CUENCA, LL., ALARCÓN, F., BOZA, A., FERNÁNDEZ-DIEGO, M., RUIZ, L., GORDO, M. L., POLER, R. y ALEMANY, M. (2015). "Rúbrica para la Evaluación de la Competencia Innovación, Creatividad y Emprendimiento en máster" en In-Red 2015 Congreso Nacional de Innovación Educativa y Docencia en Red (30 de junio y 1 de julio de 2015. València).

DE BONO, E. (1967). New think. The use of lateral thinking. New York: Basic Books.

JOHNSON, M. W., CHRISTENSEN, C. M. y KAGERMANN, H. (2008). "Reinventing your business model" en Harvard Business Review, vol. 86, issue 12, p. 50-59.

LÓPEZ, A. (2016). "Cooperación, creatividad y emprendimiento para el desarrollo de competencias. Una experiencia docente en Periodismo" en In-Red 2016 Congreso de Innovación Educativa y Docencia en Red de la Universitat Politècnica de València (7-8 de julio de 2016. València).

MARIN-GARCIA, J. A., RAMIREZ, L. y ANDREU, M. A. (2015). "Comparación de los métodos de escalas y frecuencia de comportamiento para valorar la competencia de innovación. El punto de vista de alumnos y profesor en el caso de una asignatura de máster" en In-Red 2015 Congreso Nacional de Innovación Educativa y Docencia en Red (30 de junio y 1 de julio de 2015. València).

MARTIN, B. R. y ETZKOWITZ, H. (2000). "The origin and evolution of the university species" en VEST Journal for Science and Technology Studies, vol. 13, issue 3-4, p. 9-34.

MATEU, J. M. (2015). Herramientas para la .generación y evaluación ex-ante de modelos de negocio. Tesis Doctoral. Valencia: Universitat de València, http://roderic.uv.es/handle/10550/47833

MATEU, J. M. y MARCH, I. (2016). "Searching for better business models assessment methods" en Management Decision, vol. 54, issue 10, p. 2433 - 2446.

MOSKAL, B. M. (2000). "Scoring rubrics: what, when and how?" en Practical Assessment Research \& Evaluation, vol. 7, issue 3 .

NAVAL, C. N., y CORBELLA-RUIZ, M. R. (2012). "Aproximación a la responsabilidad social universitaria: la respuesta de la Universidad a la sociedad" en Bordón. Revista de pedagogía, vol. 64, issue 3, p. 103-115.

OSTERWALDER, A. y PIGNEUR, Y. (2010). Business Model Generation. Hoboken, Nueva Jersey: John Wiley \& Sons, Inc.

PEÑA, I., GUERRERO, M. y GONZÁLEZ-PERNÍA, J. L. (2015). Global Entrepreneurship Monitor. Informe GEM España 2015. Santander: Editorial de la Universidad de Cantabria

PEREZ-ZURIAGA, A. M., LLORCA, C. y MORENO, A. T. (2015). "Desarrollo de competencias transversales y evaluación de su adquisición en el Grado de Ingeniería Civil" en In-Red 2015 Congreso Nac. de Innovación Educat. y Docencia en Red (30 de junio/1 de julio de 2015. València).

PORTER, M. E. (1985). Competitive Advantage. New York: Free Press.

(cc) EY-NC-ND 2017, Universitat Politècnica de València 
RUIZ, L., GORDO, M., FERNÁNDEZ-DIEGO, M., BOZA, A., CUENCA, LL. ALARCÓN, F. y ALEMANY-DÍAZ, M. M. (2015). "Implementación de actividades de aprendizaje y evaluación para el desarrollo de competencias genéricas: un caso práctico de aplicación de técnicas de Pensamiento de Diseño, y evaluación mediante rúbricas, de las competencias de Creatividad, Innovación y Emprendimiento" en In-Red 2015 (30 de junio y 1 de julio de 2015. València).

SHAW, S. (2007). Airline Marketing and Management (sixth edition). Hampshire, England: Ashgate

ANEXO.-

\section{CUESTIONARIO DE EVALUACIÓN EX-ANTE DE MODELOS DE NEGOCIO}

Fecha: 120

$\rightarrow$ Información estadística ${ }^{1}$ :

Nombre Edad (años):

Nivel educativo: Género $(\mathrm{H} / \mathrm{M})$ :

País de origen: Experiencia profesional (años):

¿En qué industrias (años)? $(\ldots)$

¿Has emprendido alguna vez o estás involucrado en algún emprendimiento $(\mathrm{S} / \mathrm{N})$ ?

$\rightarrow$ Equipo de trabajo en el que se ha integrado en esta Práctica:

\section{$\rightarrow$ Primer ejercicio: Evaluación intuitiva:}

0.- Por favor evalúe con $1,2,3,4$ ó 5 las expectativas de cada modelo de negocio presentado, en términos de posibilidades de éxito. Escriba para ello la letra identificativa de cada modelo de negocio presentado junto al número con el que lo evalúa. Puede haber varios modelos con la misma evaluación (junto al mismo número). Los valores de los extremos (1 y 5) aparecen descritos a continuación):

El nuevo Modelo de Negocio sería un fracaso notable en caso de implementarlo
El nuevo Modelo de Negocio cosecharía un gran éxito en caso de implementarlo

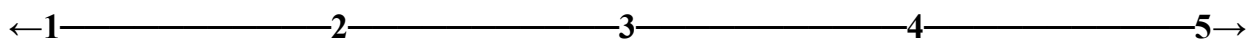

${ }^{1}$ Esta información sólo será utilizada con fines estadísticos en el marco de acciones de investigación científica. 
$\rightarrow \underline{\text { Segundo ejercicio: }}$ Este ejercicio propone evaluar cada Modelo de Negocio según una escala de ocho indicadores:

- Los cuatro primeros indicadores evalúan la fortaleza de la propuesta de valor.

- El quinto y sexto indicadores evalúan el balance entre ingresos y gastos.

- Los dos últimos indicadores (séptimo y octavo) evalúan la amenaza que suponen los competidores.

Cada indicador se presenta mediante una pregunta de evaluación y la descripción de los valores extremos. Ocasionalmente se añaden ejemplos y la descripción de los valores intermedios para facilitar la aplicación de la escala.

Cada nuevo Modelo de Negocio debe ser evaluado en cada indicador, mediante un número entero (1, 2, 3, 4 ó 5). Por favor indique la calificación escogida poniendo la letra que identifica al Modelo junto al número que indica la evaluación escogida.

1.- ¿Cómo aportará utilidad al potencial cliente la propuesta de valor incluida en nuestro modelo de negocio? ¿En qué orden de magnitud lo hará?

El modelo no aporta valor añadido o diferenciación respecto a las alternativas existentes
El modelo aporta ventajas importantes al potencial cliente, bien ahorros espectaculares de esfuerzo o dinero, bien utilidades capaces de apasionarle

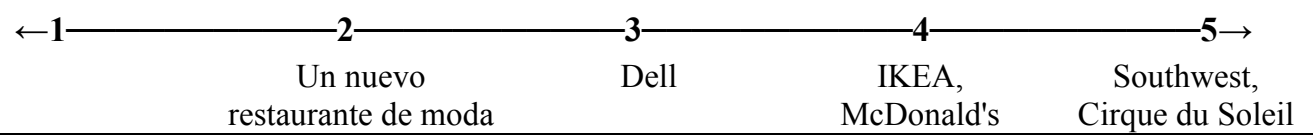

2.- ¿Están todos los elementos complementarios necesarios a disposición del cliente a un precio razonable? En caso contrario, ¿está en nuestras manos incorporarlos?, ¿en qué plazo y con qué esfuerzo?

El modelo requiere de desarrollo normativo que está fuera del alcance del emprendedor
El nuevo modelo incorpora una solución completa y no requiere ningún tipo de cambio, o bien los cambios requeridos son perfectamente entendibles por el cliente potencial

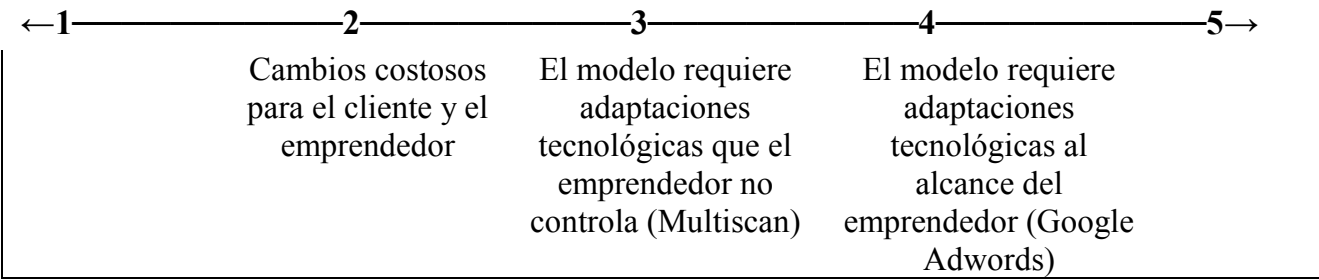


3.- ¿Cómo es de grande el segmento de potenciales clientes? ¿Qué volumen de compra representa?

\begin{tabular}{|c|c|c|}
\hline \multicolumn{2}{|l|}{$\begin{array}{l}\text { El mercado es muy reducido (local, } \\
\text { compuesto de muy pocos clientes, } \\
\text { con poca repetición y pequeñas } \\
\text { compras) }\end{array}$} & $\begin{array}{c}\text { Existe un mercado masivo glob } \\
\text { con muchos clientes potencial } \\
\text { que gastan mucho en este tipo } \\
\text { productos o servici }\end{array}$ \\
\hline$\leftarrow 1-2-$ & 3 & $-4 \longrightarrow$ \\
\hline $\begin{array}{l}\text { Mercados } \\
\text { limitados o con } \\
\text { baja tasa de } \\
\text { repetición }\end{array}$ & $\begin{array}{l}\text { Mercados } \\
\text { numerosos pero con } \\
\text { poco gasto o baja } \\
\text { repetición }\end{array}$ & $\begin{array}{l}\text { Mercados no } \\
\text { masivos pero } \\
\text { atractivos por la } \\
\text { repetición o sus } \\
\text { grandes compras }\end{array}$ \\
\hline
\end{tabular}

\section{4.- ¿Cómo de complicado resultará demostrar al potencial cliente que la propuesta le aportará valor y no se trata de una mera moda pasajera?}

El modelo representa un cambio radical en la forma en que el cliente viene haciendo las cosas y la manera de extraer el valor no resulta intuitiva
La forma de extraer valor al nuevo modelo por el cliente es evidente y no requiere mayores explicaciones, ni costes, ni esfuerzos de adaptación para el cliente

$\begin{array}{ccc}\text { Sistemas CRM } & \begin{array}{c}\text { Plataformas } \\ \text { multilaterales: } \\ \text { Google Adwords }\end{array} & \text { Southwest } \\ & \end{array}$

5.- ¿Estarán los potenciales clientes dispuestos a pagar el precio y a hacer el esfuerzo que les pedimos a cambio del valor que les ofrecemos?

\begin{tabular}{|c|c|c|c|}
\hline $\begin{array}{l}\text { Los potenciales clientes son reacios a } \\
\text { pagar por el tipo de servicios que } \\
\text { ofrece el modelo, o no están } \\
\text { dispuestos a asumir los esfuerzos que } \\
\text { se les piden }\end{array}$ & & \multicolumn{2}{|c|}{$\begin{array}{l}\text { Los potenciales clientes pagarán } \\
\text { gustosos lo que les pide el modelo y } \\
\text { harán el esfuerzo que se les pide } \\
\text { con buena predisposición }\end{array}$} \\
\hline-2 & $-3-$ & $-4-$ & $-5 \rightarrow$ \\
\hline $\begin{array}{l}\text { Clientes } \\
\text { industriales } \\
\text { atrapados en } \\
\text { inversiones } \\
\text { recientes }\end{array}$ & $\begin{array}{l}\text { Inversiones } \\
\text { importantes en } \\
\text { tecnología }\end{array}$ & $\begin{array}{l}\text { Esfuerzos } \\
\text { importantes } \\
\text { para los } \\
\text { clientes } \\
\text { (IKEA) }\end{array}$ & $\begin{array}{c}\text { Esfuerzo } \\
\text { limitado y bajos } \\
\text { precios } \\
\text { (Southwest, } \\
\text { Starbucks) }\end{array}$ \\
\hline
\end{tabular}

- ¿Será costoso ofrecer el valor prometido al cliente o contaremos por el contrario con un margen atractivo? ¿Existen mecanismos (directrices de coste) que nos permitan ampliar ese margen?

El modelo no cuenta con economías de

El modelo cuenta con economías de 


\begin{tabular}{|c|c|c|c|c|}
\hline \multicolumn{3}{|c|}{$\begin{array}{l}\text { escala u otros mecanismos que reduzcan } \\
\text { los costes unitarios proporcionando } \\
\text { márgenes atractivos, ni con efectos de } \\
\text { red u otros mecanismos que actúan como } \\
\text { motores de crecimiento }\end{array}$} & \multicolumn{2}{|c|}{$\begin{array}{r}\text { escala u otros mecanismos que reducen } \\
\text { los costes unitarios proporcionando } \\
\text { márgenes atractivos, o con efectos de red } \\
\text { u otros mecanismos que actúan como } \\
\text { motores de crecimiento }\end{array}$} \\
\hline \multicolumn{3}{|c|}{$\leftarrow 1-2-$} & $-4-$ & $-5 \rightarrow$ \\
\hline $\begin{array}{l}\text { Servicios con } \\
\text { alta } \\
\text { componente } \\
\text { de prestación } \\
\text { personal }\end{array}$ & $\begin{array}{l}\text { Economías de } \\
\text { escala sólo } \\
\text { aplicables a } \\
\text { actividades } \\
\text { corporativas o } \\
\text { asimiladas } \\
\text { (Starbucks) }\end{array}$ & $\begin{array}{l}\text { Economías de } \\
\text { escala relativas } \\
\text { en distribución } \\
\text { o fabricación } \\
\text { (Dell) }\end{array}$ & $\begin{array}{c}\text { Economías de } \\
\text { escala notables } \\
\text { y/o delegación en } \\
\text { el cliente de } \\
\text { alguna actividad } \\
\text { (Cirque du Soleil, } \\
\text { IKEA) }\end{array}$ & $\begin{array}{l}\text { Costes ínfimos } \\
\text { por alta } \\
\text { digitalización o } \\
\text { delegación casi } \\
\text { total en el } \\
\text { cliente: Google } \\
\text { Adwords }\end{array}$ \\
\hline
\end{tabular}

7.- ¿Existen propuestas de valor de competidores que resuelvan el mismo problema al cliente? ¿Cómo es el valor creado por ellas frente al creado por la nuestra? ¿Cómo de poderosos son esos competidores comparados con nosotros?

\begin{tabular}{|c|c|c|c|c|}
\hline \multicolumn{3}{|c|}{$\begin{array}{l}\text { Existe una fuerte, numerosa y } \\
\text { solvente competencia frente a la que } \\
\text { nuestro modelo no presenta unas } \\
\text { ventajas significativas }\end{array}$} & \multicolumn{2}{|c|}{$\begin{array}{r}\text { La competencia a la que nos } \\
\text { enfrentamos es escasa, débil y poco } \\
\text { solvente, y nuestro modelo es } \\
\text { claramente superior al suyo }\end{array}$} \\
\hline$-1-$ & $-2-$ & & $-4-$ & $-5 \rightarrow$ \\
\hline $\begin{array}{l}\text { Mercados } \\
\text { maduros con } \\
\text { competidores } \\
\text { más fuertes que } \\
\text { nosotros en los } \\
\text { que somos uno } \\
\text { más } \\
\end{array}$ & $\begin{array}{c}\text { Mercados maduros } \\
\text { con competidores } \\
\text { fuertes en los que } \\
\text { aportamos un } \\
\text { valor diferencial } \\
\text { relativo } \\
\text { (Starbucks) }\end{array}$ & $\begin{array}{c}\text { Mercados } \\
\text { maduros con } \\
\text { competencia } \\
\text { solvente en los } \\
\text { que aportamos } \\
\text { valor diferencial } \\
\text { (Dell) } \\
\end{array}$ & $\begin{array}{l}\text { Mercados en } \\
\text { los que } \\
\text { podemos } \\
\text { competir con } \\
\text { relativa } \\
\text { superioridad } \\
\text { (IKEA) } \\
\end{array}$ & $\begin{array}{c}\text { Mercados en } \\
\text { los que la } \\
\text { competencia no } \\
\text { existe o es } \\
\text { despreciable } \\
\text { (Cirque du } \\
\text { Soleil) } \\
\end{array}$ \\
\hline
\end{tabular}

\section{8.- ¿Tiene el modelo de negocio mecanismos para evitar la copia por parte de imitadores?}

El modelo no puede ser protegido de la copia y no incorpora mecanismo o activo específico inaccesible para la competencia y potencial competencia
El modelo dispone de mecanismos legales de protección (patentes que protegen elementos esenciales del modelo) o incorporan recursos y capacidades de difícil consecución por la competencia

\begin{tabular}{|c|c|c|c|c|}
\hline \multicolumn{5}{|c|}{$\leftarrow 1-2-$} \\
\hline $\begin{array}{l}\text { Modelos de } \\
\text { fácil } \\
\text { imitación }\end{array}$ & $\begin{array}{l}\text { Modelos de fácil } \\
\text { imitación, } \\
\text { protección } \\
\text { mediante } \\
\text { marketing } \\
\text { (Starbucks) }\end{array}$ & $\begin{array}{c}\text { Protección relativa } \\
\text { debido al volumen } \\
\text { de inversión } \\
\text { exigido para su } \\
\text { puesta en marcha } \\
\text { (Dell) }\end{array}$ & $\begin{array}{l}\text { Efectos de red o } \\
\text { similares que ayudan } \\
\text { al primer movedor a } \\
\text { mantener su ventaja } \\
\text { (Adwords) }\end{array}$ & $\begin{array}{c}\text { Protección } \\
\text { legal } \\
\text { (patentes). }\end{array}$ \\
\hline
\end{tabular}

